\title{
Detection of Vehicle's Number Plate at Nighttime using Iterative Threshold Segmentation (ITS) Algorithm
}

\author{
Maria Akther \\ Dept. of Computer Science, Daffodil International University, Dhaka, Bangladesh \\ maria-cs@diu.edu.bd \\ Md. Kaiser Ahmed \\ Dept. of CSE, United International University, Dhaka, Bangladesh \\ kaiser@cyberxp ress.net \\ Md. Zahid Hasan \\ Dept. of CSE, Green University, Dhaka, Bangladesh \\ hasan.cse@green.edu.bd
}

\begin{abstract}
An Iterative method of ind ividual nameplate detection using color images acquired from a high position is proposed for guidance of nighttime vehicles and other similar purposes. Segmentation is a very critical and difficult stage to accomplish in computer aided detection systems. Fundamentally the method contains iterative automatic thresholding and selecting the best threshold value which is applied to the original or enhanced dark night images. The main focus of the iteration based threshold to distinguish the image of the background and foreground. This method was tested on an actual outdoor vehicle images and results obtained from automatic thresholding of the experimental images are showing the validity of the method.
\end{abstract}

Index Terms - Image processing, Vehicle nameplate detection, image segmentation, Iterative segmentation method, Threshold, ITS algorithm.

\section{INTRODUCTION}

An image is a collection of spatially ordered picture elements (pixel) representing scene. Image segmentation is a fundamental yet still challenging problem in computer vision and image processing. In particular, it is an essential process for many applications such as object recognition, target tracking, content-based image retrieval and medical image processing, etc. Generally speaking, the goal of image segmentation is to partition an image into a certain number of pieces which have coherent features (color, texture, etc.) and in the mean while to group the meaningful pieces together for the convenience of perceiving [1]. Image segmentation is often used to distinguish the foreground from background. It plays an important role in image processing and pattern recognition. This process includes of dividing an image into several constituent regions [2]. Segmentation algorithms are generally based on one of two basic parts: discontinuity detection (edge based) or similarity detection (region based) [3][4][5]. In the introductory stage, many image processing and computer vision applications usually require binary images (i.e. black and white) in order to further processing. Despite the ostensible easiness of the thresholding process, it is actually a complex procedure given the vast array of occurrences and ambiences that are unique to each image.

In paper [6] they presented two techniques of segmentation algorithm (Canny edge detection and Otsu thresholding) and tested with variety of color images. These algorithms are produced binary images, which represent objects and their background which then help interpreting the content of the considered images. In the first step in image analysis, segmented an image based on discontinuity (Edge-based) or similarity detection technique (Region-based) that segments an image into regions based on based on similarity according to a predefined criteria. They presented and evaluated two methods edge based and threshold based segmentation. They proposed these two algorithms to evaluate for medical and nonmedical images. But for testing endoscopic images Canny segmentation is more suitable than Otsu's segmentation method.

A survey of image thresholding methods is conducted categorized and formulated under a uniform notation and then evaluate the performance comparison presented in paper [28]. They categorized the thresholding methods such as histogram shape, measurement space clustering, entropy, object attributes, spatial correction and local-gray level surface. They also compared 40 selected thresholding methods from various categories are compared.

The image threshold problem is acted as an important manifestation in image processing, and it not only 
reduces the image data, but also lays a good foundation for target recognition and understanding images. Character of both the local threshold segmentation and global threshold was analyzed in image segmentation. A new threshold statistic iterative arithmetic is presented to overcome the direct worth method in threshold. Threshold techniques can be categorized into two different classes: global threshold and local or adaptive threshold. In the global threshold, a single threshold value is used in an image. In the local or adaptive threshold, a threshold value is assigned to each pixel to determine whether it belongs to the foreground or the background pixel using local information around the pixel basically it groups the pixels having nearly same values. Because of the advantage of simple and easy implementation, the global threshold has been a popular technique since many years [7] [8] [9].

In this work [10] proposed an Color image segmentation algorithm based on different segmentation techniques where the background objects are recognized as the sky, ground, and trees etc based on the color and texture information. To get the advantage of simple and easy implementation of threshold techniques, they did the comparative study of Global and Local technique, clustering technique and Graph Cut method of image segmentation and tried to differentiate the background and foreground.

An application introduced in [25] Adaptive approach which is good for the selection of threshold in many occasions. This paper proposes an Iterative methodology for segmentation of traffic signal based on the prior knowledge about the size of the traffic signals. A Morphological operation is applied to s mooth the input color image in itially. A geometric area, pixel population and perimeter are used to extract the traffic signals from the input image. In this work, the input color image is divided into three channels red, green and blue and the threshold is applied to the red channel to select the red as well as amber signal and green channel is processed to select the green signal.

Image is analyzed in a range of areas such as biology, medicine, physics astronomy, geography, chemistry, meteorology and industrial manufacturing. Inside an image analysis system, segmentation has been accounted for pattern recognition and image interpretation [29]. Many different approaches for image segmentation there are; which mainly differ in the criterion used to measure the similarity of two regions and in the strategy applied to guide the segmentation process. The iterative algorithm based on the mean shift described in this paper.

Image is divided into homogenous region for color image segmentation [26]. A commonly used technique, threshold is used in this work. This technique assumes that the image presents number of components of nearly homogenous value that can separate the components by a proper choice of intensity threshold. This paper presents and efficient iterative algorith $m$ which can find the optimal thresholds value. Firstly, color image is captured from the environment and then applied the edge detection method which can detect the edge of the color images. In the second step, color image is converted into gradient map and then analyzed the regular experience values. At the last step, they were selecting the best experience value iteratively to choose the best threshold of the gradient map. They applied it the color images of cotton foreign fibers for segmenting.

Different types of technique are considered for image segmentation. Image thresholding is one of the most important techniques for image segmentation. This paper [27] presents the thresholding to find the best thresholds that minimize a weighted sum-of-squarederror function.

In this research [11] presented an adaptable segmentation method, that can be applied to different type of images, providing a better segmentation. This work is based on a model of automatic multilevel thresholding and considers techniques of group histogram quantization, analys is of the histogram slope percentage and calculation of maximum entropy to define the threshold. The technique was applied to segment the cell core and potential rejection of tissue in myocardial images of biopsies from cardiac transplant [12].

Another method analyses in [13] where automatic thresholding is used by Histogram Segmentation Using Discriminant Analysis. The most ingenuous approach is to pick up a fixed grayscale value as the threshold and classify each grayscale by checking whether it lies above or below this value. In general, the threshold should be located at the obvious and deep valley of the histogram. Especially for a well-defined image, its histogram has a deep valley between two peaks. Therefore, the optimum threshold value can be found in the valley region [14]. One extremely simple way to find a suitable threshold is to find each of the modes (local maxima) and then find the valley (minimum) between them [15]. They have proposed a novel gray level thresholding algorithm using the cluster analysis where the optimal threshold is selected on the similarity between gray levels and avoided the problem of local minima. It alleviates the multi-level thresholding problem by stopping the grouping as the expected segment number is achieved.

In point of fact, most applications of image thresholding are bounded to a particular domain where conditions are not as variable as in the normal case. Lighting conditions are one of those factors that are unpredictable and hard to model accurately. Segmentation techniques are grouped in three different classes: the first techniques named local techniques depends on the local properties of the pixels and their proximity. In next techniques an image can be segmented on the basis of information about image histogram, global texture properties. In the last techniques to obtain good segmentation results are following the split, merge and growing techniques [16] [17].

Given the aforementioned problem, this paper focuses on the analysis and evaluation of an iterative selection of 
image threshold technique to apply the nighttime images on vehicle and extracted the nameplate number accurately. The rest of the paper is organized as follows: Section II discusses the ITS materials and methods; Section III describes the algorithm of Iterative threshold selection; Section IV presents experimental results; we finally conclude in Section V.

\section{THRESHOLD BASED IMAGE SEGMENTATION}

Thresholding techniques detect a region based on the pixels value with similar intensity values. This method identify image boundary that contain solid objects on a contrast background [18]. Thresholding technique converts any format of image to gray scale image which provides a binary output image. Simultaneously this method of segmentation applies a single fixed criterion to all pixels in the image.

In global thresholding, suppose the histogram of an image composed of light objects on a dark background. The pixel intensity levels of the object and the background are grouped into two dominant models. A threshold value $\mathrm{T}$ is selected in such a way that it separates the object and the background.

$$
g(x, y)=\left\{\begin{array}{l}
1, i f q(x, y)>T \\
0, i f q(x, y)<T
\end{array}\right.
$$

Equation (1) has no indication on selecting the threshold value $\mathrm{T}$. The threshold $\mathrm{T}$ separates the object from the dark background. Any point $(x, y)$ for which $f(x$, $\mathrm{y}) \geqslant \mathrm{T}$ is called an object point. After thresholding operation, the image is segmented as follows: Pixels labeled 1 corresponds to object whereas pixels labeled 0 corresponds to the background. In global thresholding, the threshold value $\mathrm{T}$ depends only on gray levels of $\mathrm{f}(\mathrm{x}$, y).

Global thresholding technique will not produce the desired output when pixels from different segments overlap in terms of intensities [18]. The overlapping of intensities may be caused due to (a) noise (b) variation in illumination across the image. In the first case, minimum-error method can be used to estimate the underlying cluster parameters and the threshold is chosen to minimize the classification error. Variable thresholding technique is used for the latter case. Global thresholding is popular due to simplicity and easy implementation [7][4].

Whenever the background illumination is uneven, global thresholding method is not suitable. In local thresholding technique, the threshold value $\mathrm{T}$ depends on gray levels of $f(x, y)$ and some local image properties of neighboring pixels such as mean or variance. The threshold operation with a locally varying threshold function $\mathrm{T}(\mathrm{x}, \mathrm{y})$ is given by

$$
g(x, y)=\left\{\begin{array}{l}
1, \text { iff }(x, y) \geq T(x, y) \\
0, \text { iff }(x, y)<T(x, y)
\end{array}\right.
$$

$$
\text { Where } T(x, y)=f_{0}(x, y)+T_{0}
$$

$f_{0}(x, y)$ is the morphological opening of $f$, and the applied to $f_{0}$ [19]. Local thresholding is superior to the global threshold method in the case of poorly illuminated images.constant $T_{0}$ is the result of function graythresh

\section{ITERATIVE MATERIA LS AND METHODS}

Threshold is one of the widely methods used for image segmentation. It is useful for differentiating foreground from the background. The thresholding technique is divided an image directly into regions based on their intensity values and properties [20] [21].

The method is to select an adequate threshold value $\mathrm{T}$, converted binary image from grey level image [22], the advantage of getting a binary image is that it assuages the complexity of data and simplifies the process of recognition and classification.

The method is following a test function $\mathrm{T}$ and the threshold value function is computed as:

$$
T=T[x, y, p(x, y), q(x, y)]
$$

Where $\mathrm{q}(\mathrm{x}, \mathrm{y})=$ pixel value at the point $(\mathrm{x}, \mathrm{y})$

$\mathrm{P}(\mathrm{x}, \mathrm{y})=$ local property at the point $(\mathrm{x}, \mathrm{y})$

To describe in mathematically, a threshold image is termed as pixel labeling where label 1 corresponds to object and 0 corresponds to background [23].

$$
g(x, y)=\left\{\begin{array}{l}
1, i f q(x, y)>T \\
0, i f q(x, y)<T
\end{array}\right.
$$

\section{ITERATIVE THRESHOLD SELECTION (ITS) ALGORITHM}

Iterative schemes are based on the mixture of two Gaussian-based models. The basic theory behind iterative thresholding can be summarized as follows [24].

1. Choose an initial estimate of threshold $(\mathrm{T})$ which can be taken as the average of minimum and maximum intensity value.

2. Segment the image as background and foreground according to this initial threshold.

$$
\begin{aligned}
& \text { a) } G_{1}=\{f(x, y): f(x, y)>T\} \\
& \text { b) } G_{2}=\{f(x, y): f(x, y)<=T\}
\end{aligned}
$$

3. Compute the average intensity values of each set, let $m_{1}$ are the average of $G_{1}$ and $m_{2}$ be the average of $\mathrm{G}_{2}$.

4. A new threshold, $\mathrm{T}$ ' is calculated as follows:

$T=\frac{m_{1}+m_{2}}{2}$ 
5. Repeat step 2 until the difference between $\mathrm{T}$ and $\mathrm{T}$ ' is minimal.

In positive realization of the initial switch function will enter one by one image into foreground and background, in the first pass after the end of the image scanning, the average value of two integrators to determine a threshold. Threshold control switch again will enter into foreground and background, and used as a new switching function. Iterative and so forth until the switching function is not changed, this time to get the foreground and background shall be final segmentation results.

\section{A. Diagrammatic Representation of ITS}

Diagrammatic representation of Iterative Selection Method (ITS) is shown in Figure. 1.

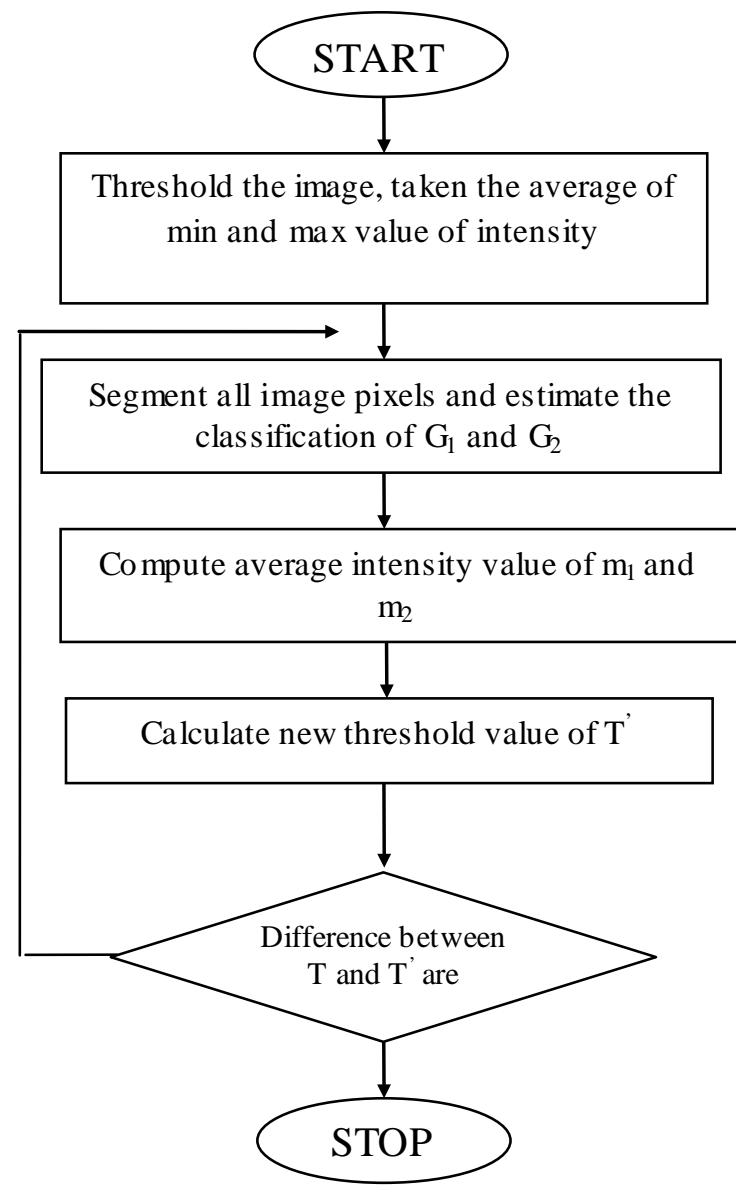

Figure 1. Diagrammatic representation of Iterative Threshold Selection Method

As shown in Figure 1, in first step taken the minimum and maximu $\mathrm{m}$ value of intensity image and calculate the average of minimum and maximum intensity values to select the threshold.

In second step, all image pixels are segmented and estimated based on the classification of background and foreground according to this initial threshold of using equation 6 and 7.

In third step, again calculate the average intensity values for each set where $\mathrm{m} 1$ denotes the average of G1 and $\mathrm{m} 2$ denotes the average of $\mathrm{m} 2$.
In fourth step, calculate the new threshold value based on the average of $\mathrm{m} 1$ and $\mathrm{m} 2$ set.

In last step, it will check the minimal difference between old threshold and new threshold. The step will be repeated until it will reach the minimal difference.

\section{EXPERIMENT SIMULATION AND RESULT ANALYSIS}

To evaluate the performance of the iterative threshold selection (ITS) algorithm, a simulation study is carried out. In this study, images of some scenes are generated and then segmented by the ITS algorithm. Three different images have size of 400 X 300, 244 X 207 and 259 X 194 pixels. And the grey level value of each pixel is quantized to 8 bits, i.e. from $0-255$. All the investigations are carried out in an Intel compatible personal computer with $2.27 \mathrm{GHz}$ corei5 CPU.

The purpose of the segmentation in this system is extracting regions from images. In Figure. 2(a) shows the original images in light parking areas and besides that in Figure. 2(b), no light at midnight in the same place. We extract the nameplate number after applying the ITS algorithm (in Figure 2(b)) and the resultant image is shown in Figure 2(c). The object was successfully separated from its background. In Figure 2(c), it can be observed that the white (1) represents the foreground and black (0) represents the background.

The purpose of the segmentation in this system is extracting regions from images. In Figure. 2(a) shows the original images in light parking areas and besides that in Figure. 2(b), no light at midnight in the same place. We extract the nameplate number after applying the ITS algorithm (in Figure 2(b)) and the resultant image is shown in Figure 2(c). The object was successfully separated from its background. In Figure 2(c), it can be observed that the white (1) represents the foreground and black (0) represents the background.

For the Figure 3(a) above, the image with Gaussian Noise was first converted to grayscale then global thresholding using iterative algorith $\mathrm{m}$ was performed on the image. 


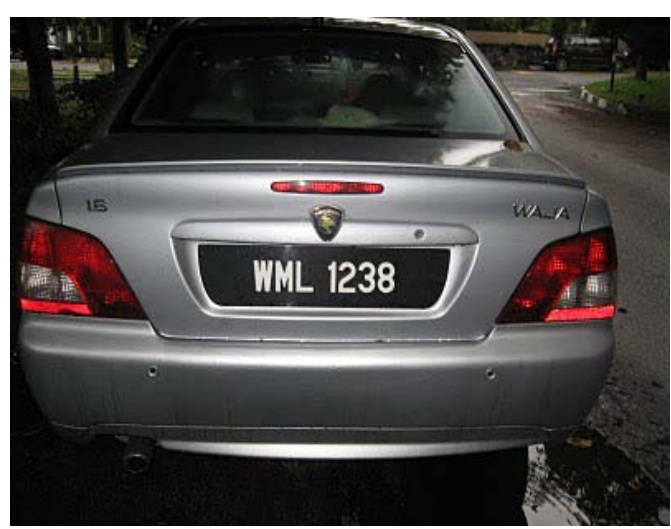

(a) Original Image

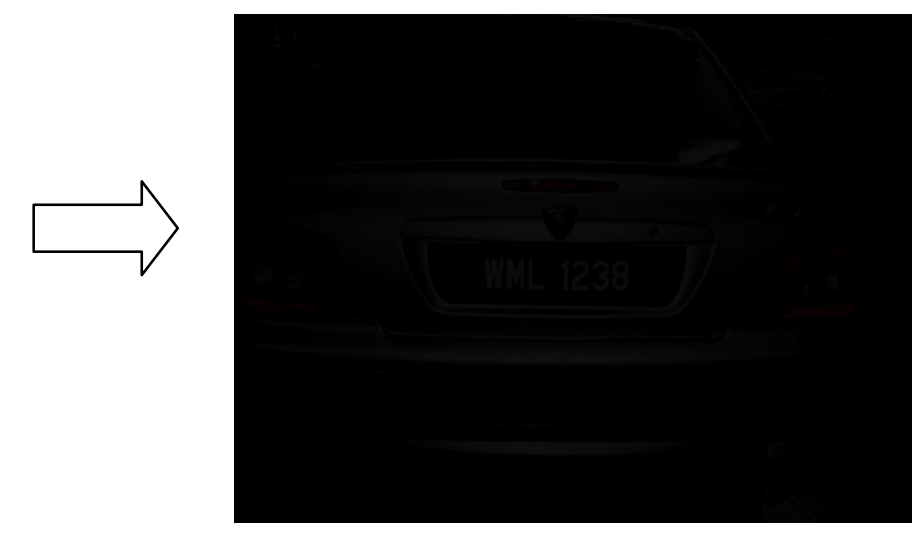

(b) No light at midnight

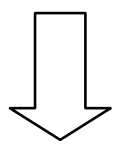

Figure 1

File Edit View Insert Tools Desktop Window Help

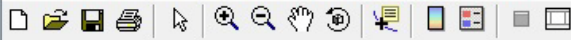

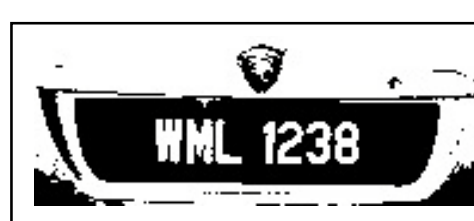

(c) After applying ITS algorithm

Figure 2. Results of the steps of the ITS algorithm (a) original image (400 X 300) (b) No Light Image at midnight, the results of (c) Threshold Image extracting the number plate. 


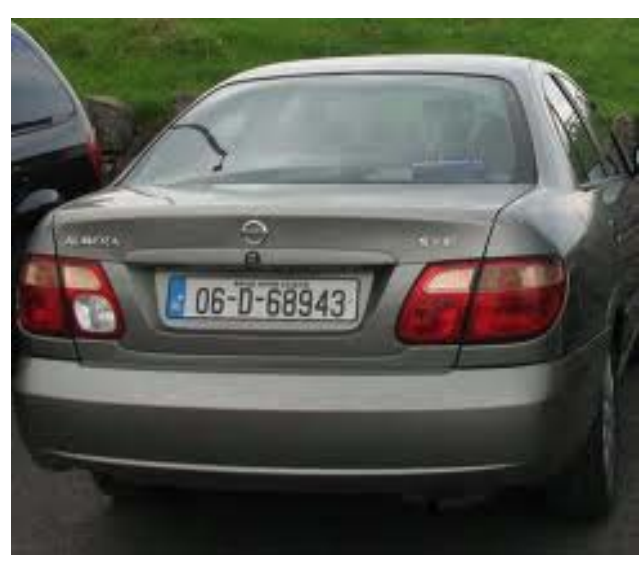

(a) Original Image

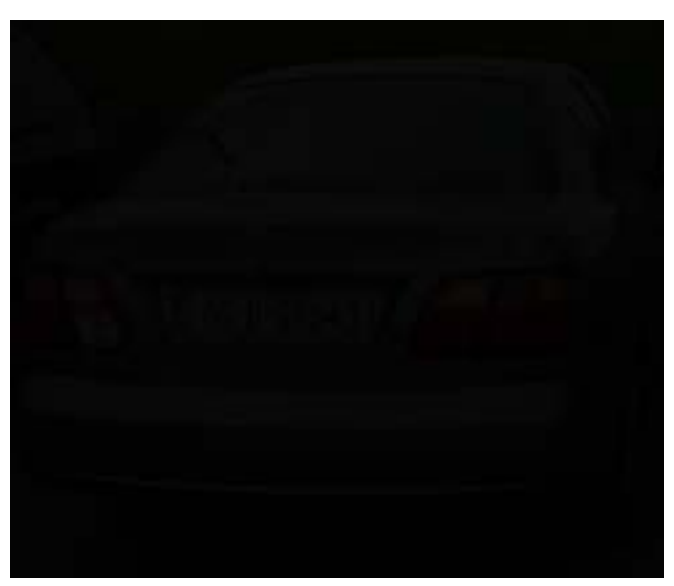

(b) No light at midnight
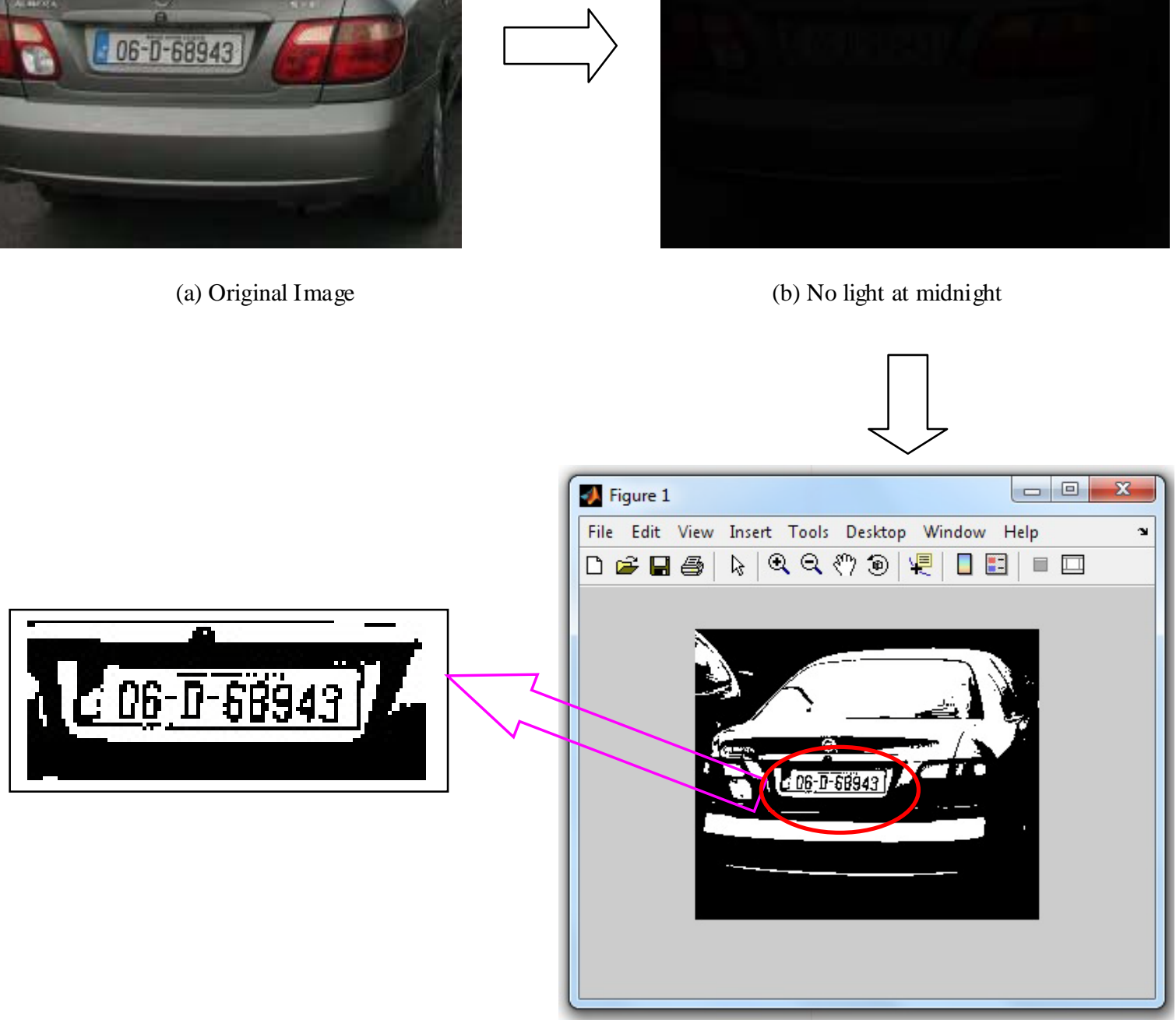

(c) After applying ITS algorithm

Figure 3. Results of the steps of the ITS algorithm (a) original image (244 X 207) (b) No Light Image at midnight, the results of (c) Threshold Image extracting the number plate.

To assess the performance of the ITS algorithm on real-life images, some practical images are segmented. Good segmentation results are also obtainable for real- life images. In general this algorithm is worthy of consideration for segmenting images where long computation time can be tolerated. 


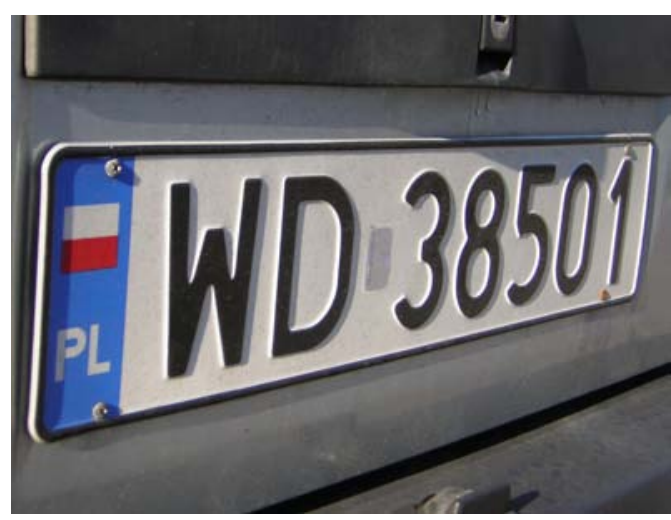

(a) Original Image

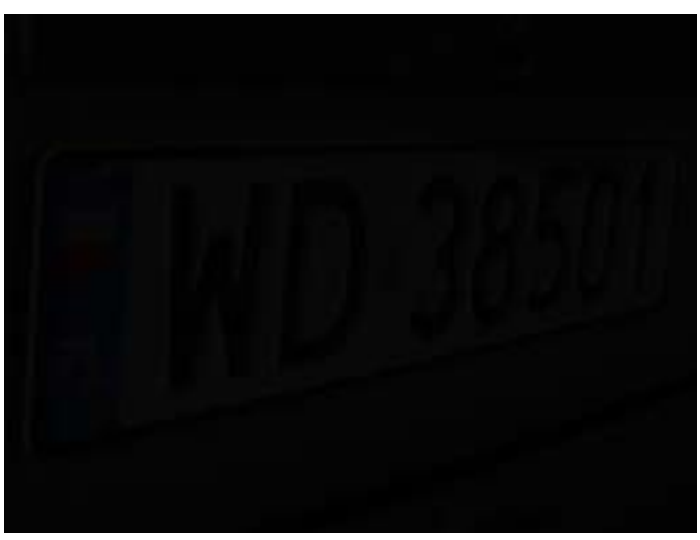

(b) No light at midnight

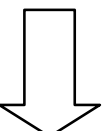

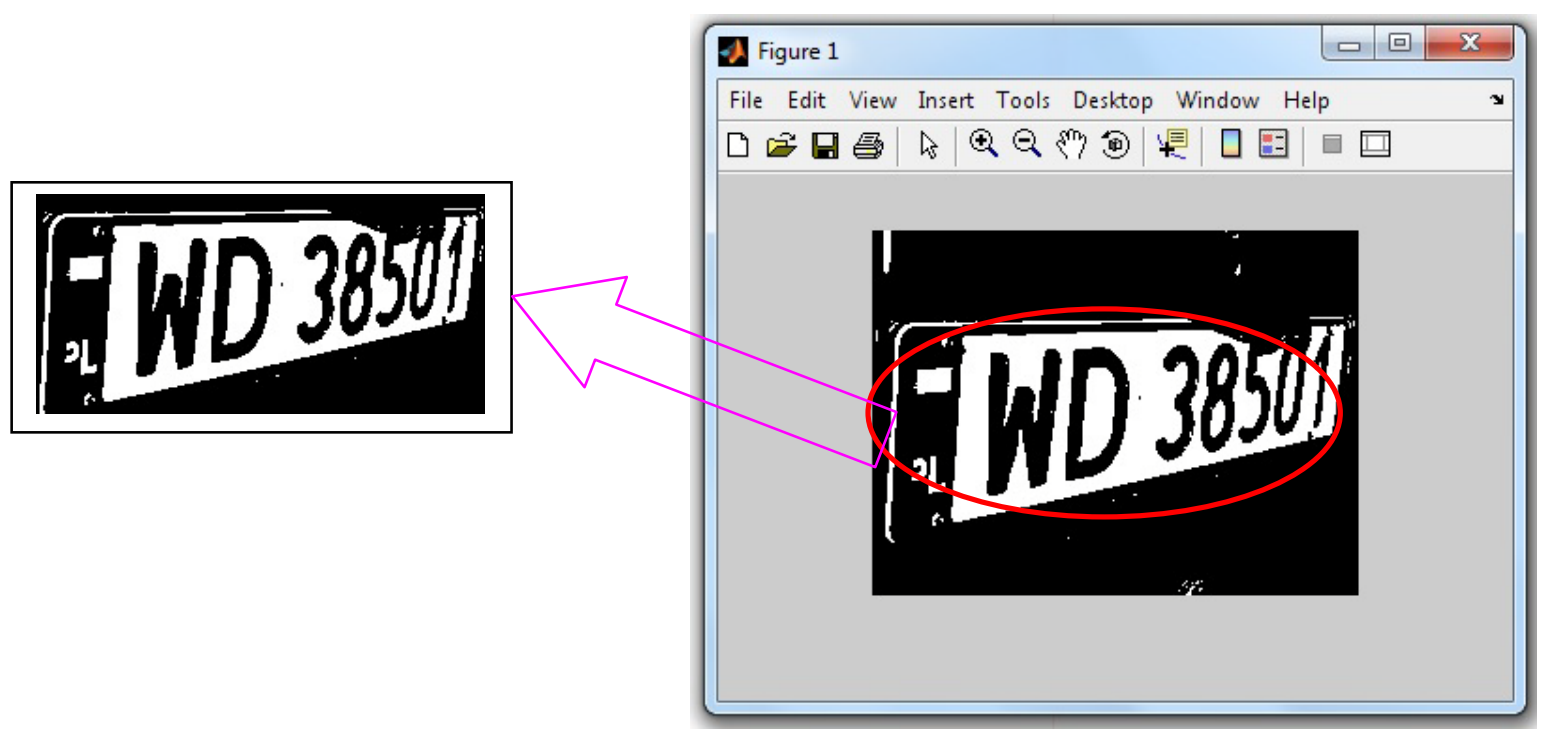

(c) After applying ITS algorithm

Figure 4. Results of the steps of the ITS algorithm (a) original image (259 X 194) (b) No Light Image at midnight, the results of (c) Threshold Image extracting the number plate.

To evaluate the performance of the ITS algorith $\mathrm{m}$, we apply three car in a parking place images as shown in Figure. 2(b), 3(b) and 4(b) respectively.

From these experimental results, we conclude that the ITS method provides better performance and accurately retrieve the nameplate information from a dark image.

\section{CONCLUSION}

This work presents a computationally efficient method designed for automatic segmentation of a grey level thresholding algorithm using the cluster analysis for detecting the entire image and then retrieves the nameplate number for each image. The optimal threshold is determined based on the similarity between grey levels. Results obtained from automatic thresholding of the experimental images are showing the validity of the method. The main area of the iterationbased threshold to distinguish the image of the foreground and background where, but in the detail of the image is not very good distinction. Certain images, small changes in the data can cause the segmentation huge change, just a slight change in the data of the two, but the effect of segmentation has a great contrast. The use of split, merge and growing techniques would be an interesting addition to explore as proposed for future work.

\section{REFERENCES}

[1] D.A. Forsyth and J. Ponce, Computer Vision: A Modern Approach, Prentice Hall, 2002. 
[2] Agus Zainal Arifin and Akira Asano, "Image Segmentation by Histogram Thresholding using Hierarchical Cluster Analysis”, Journal on Pattern Recognition Letters, Vol. 27 Issue 13, Pages 15151521, 2006.

[3] Jaskirat Kaur, Sunil Agarwal,Renu Vig, “A Comparative analysis of Thresholding and Edge detection segmentation Techniques”, International Journal of Computer Applications, Volume 39,No. 15, Feb. 2012.

[4] A. L. Amri Salem Saleh, N. V. Kalyankar, S. D. Khamitkar ,’Image Segmentation By Using Thresholding Techniques”, Journal Of Computing, Volume 2,Issue 5, pp. 83-86, 2010.

[5] A.S. Abutaleb, “ Automatic Thresholding of GrayLevel Pictures Using Two Dimensional Entropy”, Computer Vision, Graphics and Image Processing, Vol.47, pp. 22-32, 1989.

[6] Jamil A. M. Saif, A li Abdo Mohammed Al-Kubati, Abdultawab Saif Hazaa, Mohammed Al-Moraish, Image Segmentation Using Edge Detection and Thresholding, The 13th international Arab Conference on information technology ACIT, pp. 473-476, 2012.

[7] A.S. Abutaleb, "Automatic Thresholding of GrayLevel Pictures Using Two Dimensional Entropy”, Computer Vision, Graphics, and Image Processing, Vol.47, PP.22-32, 1989.

[8] J. Kittler and J. Illingworth, "Minimum Error Thresholding”, Pattern Recognition, Vol.19, No.1, PP.41-47, 1986.

[9] K.H. Liang and J.J.W Mao, "Image Thresholding by Minimizing the Measures of Fuzziness”, Pattern Recognition, Vol.28, No.1, PP.41-51, 1995.

[10] Purnashti Bhosale and Aniket Gokhale, Segmentation of Color Images Based on Different Segmentation Techniques, International Journal of Electronics and Computer Science Engineering, Vol.2, No.2, pp. 640-644, 2013.

[11] Pritee Gupta, Vandana Malik and Mallika Gandhi, Implementation of Multilevel Threshold Method for Digital Images Used In Medical Image Processing, International Journal of Advanced Research in Computer Science and Software Engineering, Vol.2 Issue 2, 2012.

[12] A. N. Otsu, "A threshold selection method from gray-level histogram”. IEEE Transactions on Systems, Man. And Cybernetics, pp. 62-66, 1978.

[13] Agus Zainal Arifin and Akira Asano, Image Thresholding by Histogram Segmentation Using Discriminant Analysis, In Proceedings of Indonesia-Japan Joint Scientific Symposium, IJJSS,pp. 169-174, 2004.

[14] O. J. Tobias and R. Seara, “Image segmentation by histogram Trans. On Image Processing, vol. 11, pp. 1457-1465, 2002.

[15] R. C. Gonzalez and R. E. Woods, Digital Image Processing. Addison-Wesley Publishing Company, 1993.

[16] Gonzalez and Woods, "Digital image processing", 2nd Edition, prentice hall, 2002,May 2010.

[17] Kenneth R. Castelman, "Dig ital image processing", Tsinghua Univ, Press, 2003.

[18] S. Jayaraman, S. Esakkirajan, T. Veerakumar, Digital Image Processing, Tata McGraw Hill Education Private Limited, 2009.

[19] R. C. Gonzalez and R. E. Woods, Digital Image Processing (Prentice Hall, 2002).

[20] Sheenam Bansal and Ramn Maini, “A Comparative Analysis of Iterative and Ostu's Thresholding Techniques", International journal of computer applications, vol 66, No.12, pp. 45-47, 2013.

[21] Otsu, N., "A threshold selection method from graylevel histograms", IEEE trans. Syst. Mancybern., SMC-9, pp. 62-66, 1979.

[22] Rafel C. Gonzalez, Richard E. Woods,”Digital Image Processing”, Upper Saddle River, NJ, Prentice Hall, 2001.

[23] O. Nina, B. S. Morse, and W. A. Barrett, "A recursive Otsu thresholding method for scanned document binarization," IEEE Workshop on Applications of Computer Vision (WACV), pp. 307-314, January 2011.

[24] R.T.W. Calvard et al. "Picture thresholding using an iterative selection method", IEEE Transactions on Systems Man and Cybernetics, pp. 203-216, 1978.

[25] Vanniappan Balamurugan and Senthamarai Kannan, "Detection of traffic signal by adaptive approach and shape constraints", The International Arab Journal of Information Technology, Vol. 8, No. 4,pp. 345-349, October 2011.

[26] Xin Zhang, Daoliang Li, Wenzhu Yang, Jinxing Wang, Shuangxi Liu, "An Efficient Iterative Thresholding Algorithms for Color Images of Cotton Foreign Fibers", Computer and Computing Technologies in Agriculture IV, IFIP Advances in Information and Communication Technology Volume 347, 2011, pp 710-719, October 2010.

[27] Liju Dong, Ge Yu, "An Efficient Iterative Optimization Algorithm for Image Thresholding", Computational and Information Science Lecture Notes in Computer Science Volume 3314, pp 1079-1085, 2005.

[28] Mehmet Sezgin and Bulent Sankur"Survey over image thresholding techniques and quantitative performance evaluation", Journal of Electronic Imaging, pp. 146-165, January 2004.

[29] Roberto Rodríguez Morales, Didier Domínguez, Esley Torres and Juan H. Sossa, "Image Segmentation Through an Iterative Algorithm of the Mean Shift ", book chapter, October 24, 2012. 


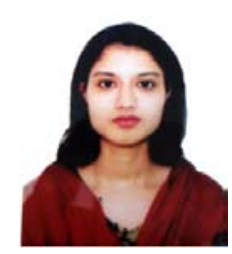

development,

Maria Akther, has completed her B.Sc.(honors) in Computer Science from Daffodil International University, Dhaka, Bangladesh. She is working as a Software Developer at CyberXpress.Net. Her research interest includes large-scale software Computational Biology, biological data management, computational genomics/sequence analysis, structural biology and image processing.

Md. Kaiser Ahmed, is a M.Sc. Student of Department of Computer Science and Engineering, Faculty of Engineering, United International University, Dhaka, Bangladesh and also working as a Senior Programmer at CyberXpress.Net. He received his B.Sc. degree in Mechanical Engineering from Khulna University of Engineering and Technology. His main research interests include formal methods and automated verification, large-scale software development, requirements analysis in Software Eng ineering fields.

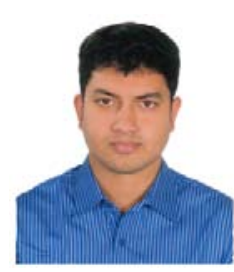

Md. Zahid Hasan, is working as a Lecturer in the Department of Computer Science and Eng ineering at Green University, Bangladesh. He received his B.Sc. (Hons)and M.Sc. degree from the Department of Information and Communication Engineering, University of Rajshahi in 2007 and 2009 respectively. His research interest include Long Term Evolution (LTE), MIMO-OFDM/ OFDMA, Signal Processing and Image Processing. 